\title{
Heavy-flavour jet measurements with ALICE
}

\author{
Marianna Mazzilli for the ALICE Collaboration*广 \\ Dipartimento Interateneo di Fisica 'M.Merlin' and Sezione INFN, Bari, Italy \\ E-mail: marianna.mazzilliecern.ch
}

\begin{abstract}
Heavy-flavour quarks, due to their large masses, are produced in the early stages of the relativistic hadronic collisions via hard scattering processes. Therefore, they are ideal probes to investigate the properties of the Quark-Gluon Plasma (QGP) produced in ultra-relativistic heavy-ion collisions, since they experience the full system evolution. In this contribution, the latest results on heavy-flavour jet production measured with the ALICE detector in $\mathrm{pp}$ and $\mathrm{p}-\mathrm{Pb}$ collisions from the LHC Run 2 data are reported. In particular, measurements of heavy-flavour decay-electron jets in pp collisions at $\sqrt{s}=5.02 \mathrm{TeV}$ and in $\mathrm{p}-\mathrm{Pb}$ collisions at $\sqrt{s_{\mathrm{NN}}}=5.02 \mathrm{TeV}$ as well as of $\mathrm{D}$-meson tagged jet in pp collisions at $\sqrt{s}=5.02,7 \mathrm{TeV}$ and in $\mathrm{p}-\mathrm{Pb}$ collisions at $\sqrt{s_{\mathrm{NN}}}=5.02$ $\mathrm{TeV}$ are presented. The latter includes also studies on the jet momentum fraction carried by the $\mathrm{D}$ meson. Moreover, a characterisation of the spatial profile and of the internal composition of the charm jet by means of the azimuthal correlations of D-mesons with charged particles in pp and in $\mathrm{p}-\mathrm{Pb}$ collisions at center-of-mass energy of $5.02 \mathrm{TeV}$ is reported.
\end{abstract}

7th Annual Conference on Large Hadron Collider Physics - LHCP2019

20-25 May, 2019

Puebla, Mexico

\footnotetext{
${ }^{*}$ Speaker.

†This work was supported by 'A. della Riccia' foundation fellowship.
} 


\section{Introduction}

The measurement of heavy-flavour jets gives more direct access to the initial parton kinematics and can provide further constraints on heavy-quark energy loss, in particular adding information on how the radiated energy is dissipated in the medium. Studies in pp collisions are mandatory to characterise heavy-quark production and fragmentation in vacuum, constituting the natural reference for interpreting heavy-ion collision results. Differences between results from $\mathrm{pp}$ and $\mathrm{p}-\mathrm{Pb}$ collisions can give insight on how the heavy-quark production and hadronisation into jets is affected by cold nuclear matter effects. The study of angular correlations between heavy-flavour particles and charged particles allows us to characterize the heavy-quark fragmentation process and its possible modification in a hot nuclear matter environment. Moreover, the study of the near-side correlation peak angular profile through the azimuthal-correlation distributions of D mesons with charged particles allows for a detailed characterisation of charm-induced jets, in terms of the charged particle multiplicity and constituent transverse-momentum distribution.

\section{Results}

\subsection{Heavy-flavour decay-electron tagged jets}

The ALICE Collaboration measured the cross section of heavy-flavour decay-electron tagged jets at mid-rapidity in pp and in $\mathrm{p}-\mathrm{Pb}$ collisions at center-of-mass energy of $5.02 \mathrm{TeV}$. With the ALICE detector [1,2], the heavy-flavour decay-electrons, i.e. the tagging particles, were selected by subtracting from the inclusive electron sample the contribution of background electrons in the transverse momentum $\left(p_{\mathrm{T}}\right)$ range $4<p_{\mathrm{T}}^{e}<18 \mathrm{GeV} / c$. The main background sources come from the photon conversions $\left(\gamma \rightarrow e^{+} e^{-}\right)$in the beam pipe and in the material of the innermost layers of the Inner Tracking System (ITS) and Dalitz decays of neutral mesons $\left(\pi^{0} \rightarrow \gamma e^{+} e^{-}\right.$and $\eta \rightarrow$ $\gamma e^{+} e^{-}$) [3]. Heavy-flavour decay-electron jets were reconstructed in the range of $10<p_{\mathrm{T}, \mathrm{jet}}^{\mathrm{ch}}<$ $60 \mathrm{GeV} / c$ using tracks of charged particles (track-based jets) with the anti- $k_{\mathrm{T}}$ algorithm [4], as implemented in the FastJet [5] software package. The $p_{\mathrm{T}}$-differential cross section was corrected for inefficiencies in the reconstruction and selection of the tagging particles as well as of the charged tracks. Additionally, the average background deriving from the underlying events was extracted using the Random Cone method [6] and then subtracted. Finally, an unfolding technique was applied to account for the limited detector transverse momentum resolution on the jet reconstruction and for the background fluctuations. The measurement was performed with various values of the resolution parameter: $R=0.3,0.4$ and 0.6 . The resulting $p_{\mathrm{T}}$-differential cross section of heavyflavour decay-electron tagged jets in $\mathrm{p}-\mathrm{Pb}$ collisions at $\sqrt{s_{\mathrm{NN}}}=5.02 \mathrm{TeV}$ is reported in the left panel of Fig. 1. The measurement is compared with Next-to-Leading Order pQCD calculations obtained with POWHEG [7] coupled with PYTHIA 6[8] for the generation of the parton shower and the hadronization treatment. A good agreement between data and model predictions is observed in $\mathrm{p}-\mathrm{Pb}$ collisions; the same conclusion is extracted also from the comparison of models and data in pp collisions. In order to further investigate the possible presence of cold nuclear matter effects on heavy-flavour jet production, the nuclear modification factor $\left(R_{\mathrm{pPb}}\right)$, defined as the ratio between the cross section in $\mathrm{p}-\mathrm{Pb}$ collisions and in $\mathrm{pp}$ collisions (scaled by the mass number $A$ ), was computed. As shown in the right panel of Fig. 1, the $R_{\mathrm{pPb}}$ with different values of the resolution parameters 

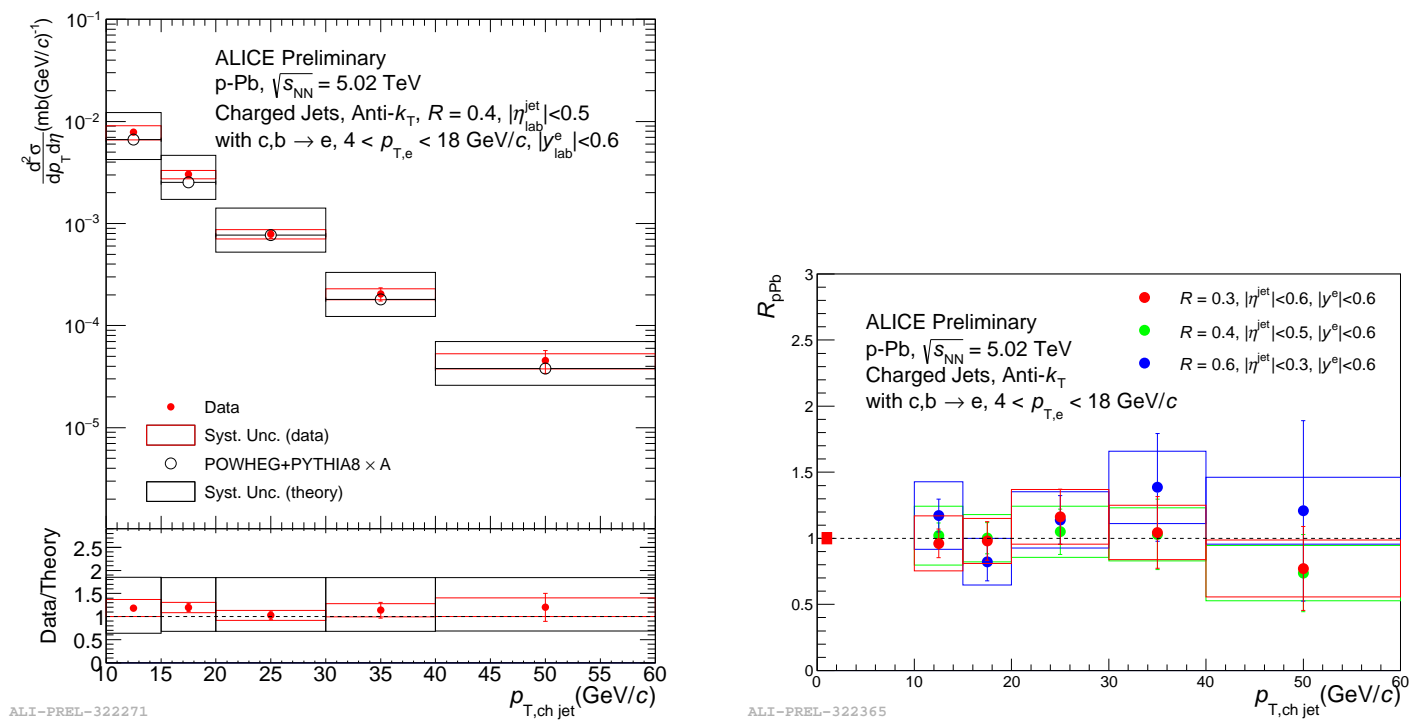

Figure 1: Left: $p_{\mathrm{T}}$-differential cross section of heavy-flavour decay electrons in pp collisions at $\sqrt{s}=5.02$ $\mathrm{TeV}$ with $10<p_{\mathrm{T}, \mathrm{jet}}^{\mathrm{ch}}<60 \mathrm{GeV} / c$ for $R=0.4$. Right: $R_{\mathrm{pPb}}$ of heavy-flavour decay-electron jet in $\mathrm{p}-\mathrm{Pb}$ collisions with $10<p_{\mathrm{T}, \mathrm{jet}}^{\mathrm{ch}}<60 \mathrm{GeV} / c$ with different values of the resolution parameter $R$.

are consistent within uncertainties and compatible with unity, suggesting a limited impact of cold nuclear matter effects.

\subsection{D-meson tagged jets}

The production of charm jets in pp collisions at $\sqrt{s}=5.02$ and $7 \mathrm{TeV}$ [9] and in $\mathrm{p}-\mathrm{Pb}$ collisions at $\sqrt{s_{\mathrm{NN}}}=5.02 \mathrm{TeV}$ was measured with ALICE. Charm jets were identified by the presence of a prompt $\mathrm{D}^{0}$ meson among their constituents. The $\mathrm{D}^{0}$ mesons are reconstructed via their hadronic decay $\mathrm{D}^{0} \rightarrow \mathrm{K}^{-} \pi^{+}$in $3<p_{\mathrm{T}}{ }^{\mathrm{D}}<36 \mathrm{GeV} / c$. The $\mathrm{D}^{0}$-meson tagged jets reconstruction was performed using the track-based procedure with the FastJet [5] anti- $k_{\mathrm{T}}$ algorithm [4], with a resolution parameter $R=0.3$ and 0.4 , in the range of $5<p_{\mathrm{T}, \text { jet }}^{\mathrm{ch}}<50 \mathrm{GeV} / \mathrm{c}$. The $p_{\mathrm{T}}$-differential cross section of $\mathrm{D}^{0}$-tagged charm jets was corrected for the reconstruction efficiency, acceptance, b-hadron feed-down contribution and unfolded for the finite detection of the jet momentum resolution. In addition, in the measurements in $\mathrm{p}-\mathrm{Pb}$ collisions, the background coming from the underlying event was subtracted on a jet-by-jet basis prior to the unfolding. The left panel of Fig. 2 shows the comparison of the $p_{\mathrm{T}}$-differential cross section of $\mathrm{D}^{0}$-meson tagged charged-particle jets in pp and in $\mathrm{p}-\mathrm{Pb}$ collisions at center-of-mass energy of $5.02 \mathrm{TeV}$. The consistency of the results among the two collision systems suggests that the modification of charm jets is not significantly affected by the cold nuclear matter effects as well as by possible final-state effects. Additionally, the $R_{\mathrm{pPb}}$ of D-meson jets was computed and it was found to be compatible with unity within the uncertainties, suggesting a small impact of cold nuclear matter effects on charm quark production.

In order to characterise the heavy quark production and fragmentation in more details, the distribution of the jet momentum fraction carried by $\mathrm{D}^{0}$ mesons in the direction of the jet axis 

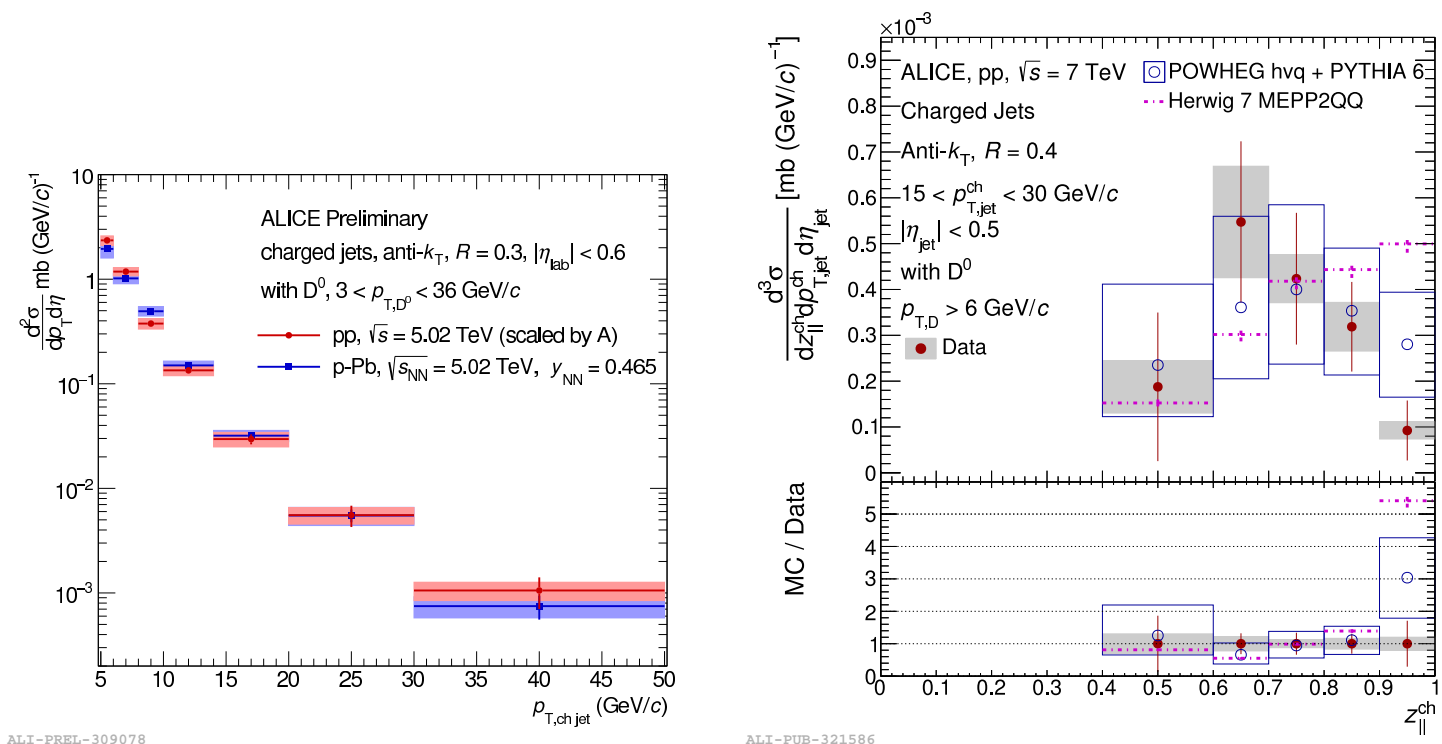

Figure 2: Left: $p_{\mathrm{T}}$-differential cross section of $\mathrm{D}^{0}$ meson tagged jets in $\mathrm{pp}$ (red) and $\mathrm{p}-\mathrm{Pb}$ (blue) collisions at center-of-mass energy of $5.02 \mathrm{TeV}$ with $5<p_{\mathrm{T} \text {,jet }}^{\text {ch }}<30 \mathrm{GeV} / c$. Right: $z_{\|, D}^{\text {ch }}$-differential cross section of $\mathrm{D}^{0}$ meson tagged jets in pp collisions at $\sqrt{s}=7 \mathrm{TeV}$ with $5<p_{\mathrm{T}, \text { jet }}^{\text {ch }}<15 \mathrm{GeV} / \mathrm{c}$.

$z_{\|, D}^{\text {ch jet }}=\frac{\vec{p}_{\text {ch jet }} \cdot \vec{p}_{D}}{\vec{p}_{\text {ch jet }} \vec{p}_{\text {ch jet }}}$ was extracted in pp collisions at $\sqrt{s}=7 \mathrm{TeV}$. In the right panel of the Fig. 2 the $z_{\|, D}^{\text {ch }}$-differential cross section of $\mathrm{D}^{0}$-meson tagged jets for $5<p_{\mathrm{T}, \text { jet }}^{\text {ch }}<15 \mathrm{GeV} / c$ is reported. Both the $z_{\|, \mathrm{D}}^{\text {ch jet }}$ and the $p_{\mathrm{T}}$-differential cross section of D-meson jets are well described by the Next-to-Leading-Order pQCD calculations of POWHEG coupled with PYTHIA6 shower MC predictions $[7,8]$.

\subsection{D-meson azimuthal correlations with charged particles}

A complementary characterisation of the charm jets was performed with ALICE by studying the angular-correlations of prompt $\mathrm{D}$ mesons $\left(\mathrm{D}^{0}, \mathrm{D}^{*+}\right.$ and $\left.\mathrm{D}^{+}\right)$with charged particles in pp and in $\mathrm{p}-\mathrm{Pb}$ collisions at center-of-mass energy of $5.02 \mathrm{TeV}$. As a general feature, the azimuthal correlation distributions present a "near-side" peak at $(\Delta \varphi, \Delta \eta)=(0,0)$ and an "away-side" peak at $\Delta \varphi=\pi$ extending over a wide pseudorapidity range. Such measurement allows to probe the internal composition and spatial profile of charm jets by evaluating the near-side peak angular profile, charged particle multiplicity and constituent $p_{\mathrm{T}}$ distribution. Moreover, studies as a function of the charged-particle $p_{\mathrm{T}}$ can provide insight into how the jet-momentum fraction not belonging to the $\mathrm{D}$ meson is redistributed among the other particles produced by the parton fragmentation, as well as on the correlations between the $p_{\mathrm{T}}$ of those particles and their radial displacement from the jet axis, closely related to the width of the near-side correlation peak. In order to study the properties of the D-meson correlations with charged particles, the azimuthal-correlation distributions $\Delta \varphi(\Delta \varphi=$ $\left|\varphi_{\mathrm{ch}}-\varphi_{\mathrm{D}}\right|$ within $|\Delta \eta|<1$ ) were fitted with a function composed of two Gaussian terms to describe the near- and away-side peaks, induced by the jet containing the $\mathrm{D}$ meson and the recoiling jet, and a constant term to describe the pedestal of the distribution, determined mainly by correlations with 


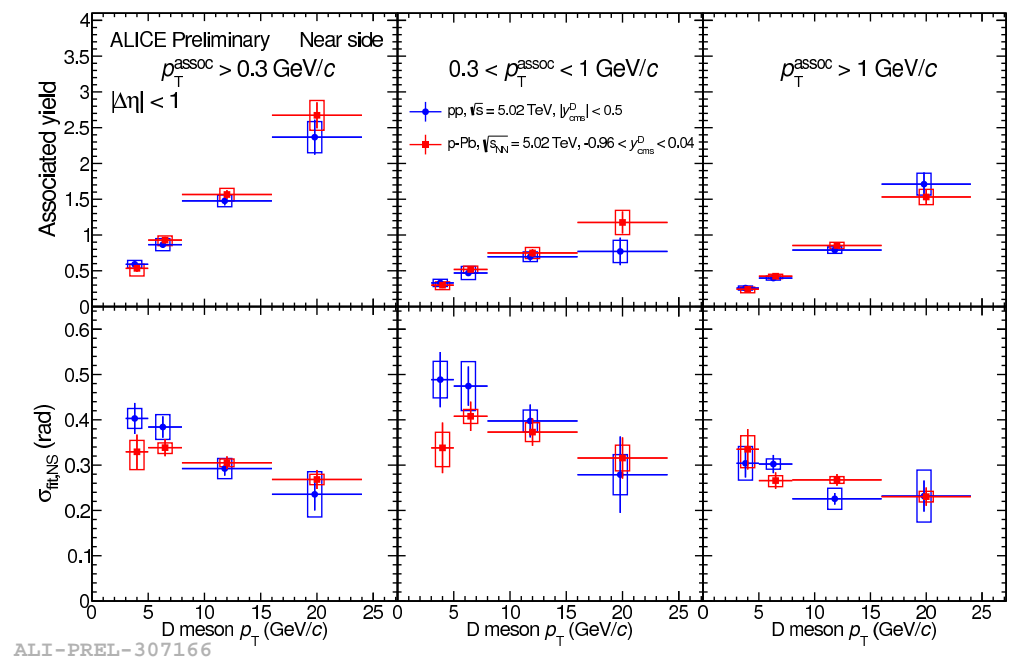

Figure 3: Comparison of near-side yields (top) and widths (bottom) in pp and in $\mathrm{p}-\mathrm{Pb}$ collisions at centerof-mass energy of $5.02 \mathrm{TeV}$ as a function of D-meson $p_{\mathrm{T}}$ in different $p_{\mathrm{T}}^{\text {assoc }}$ intervals.

particles from the underlying event. A detailed description of the analysis procedure can be found in [10]. In Fig. 3 the associated yields and the widths $\left(\sigma_{\text {fit,NS }}\right)$ of the near-side peak $(\Delta \varphi \sim 0)$ are shown as a function of the D-meson $p_{\mathrm{T}}$ for different $p_{\mathrm{T}}$ intervals of the associated tracks $\left(p_{\mathrm{T}}^{\text {assoc }}\right)$ in $\mathrm{pp}$ and $\mathrm{p}-\mathrm{Pb}$ collisions at center-of-mass energy of $5.02 \mathrm{TeV}$. The results are compatible between the two collision systems within the current precision of the measurements. In particular, an increase of the height of the near-side correlation peak, corresponding to a higher number of particles produced in the fragmentation of the charm quark, is observed for increasing values of the D-meson $p_{\mathrm{T}}$. A decrease of the peak width for increasing $p_{\mathrm{T}}{ }^{\mathrm{D}}$ is also observed, related to an increased near-side peak collimation. Moreover, the near-side observables were compared to predictions from PYTHIA6 [8] (with several Perugia tunes), PYTHIA8 [11] and POWHEG coupled with PYTHIA6 [7]. An overall good agreement of simulations was found in most of the kinematic ranges, as shown in the Fig. 4.

\section{Summary}

The $p_{\mathrm{T}}$-differential production cross-sections of heavy-flavour decay-electron tagged jets and of $\mathrm{D}$-meson tagged charged jets were measured in $\mathrm{pp}$ and $\mathrm{p}-\mathrm{Pb}$ collisions, and the jet momentum fraction carried by the $\mathrm{D}^{0}$ meson was extracted in pp collisions in two $p_{\mathrm{T}, \text { jet }}^{\mathrm{ch}}$ ranges. The measurements were in agreement with POWHEG coupled with PYTHIA6 predictions. Additionally, the $R_{\mathrm{pPb}}$ for heavy-flavour electron jets and $\mathrm{D}^{0}$-meson jets were measured and their compatibility with unity suggests that the production of jets from heavy-quarks is not significantly affected by the cold nuclear matter effects as well as by possible final-state effects. A detailed characterisation of the spatial profile and the internal composition of charm jets was performed by exploiting the azimuthal correlation distributions of $\mathrm{D}$ mesons with charged particles in $\mathrm{pp}$ and in $\mathrm{p}-\mathrm{Pb}$ collisions. The compatibility of the results between the two collision systems does not suggest the presence 


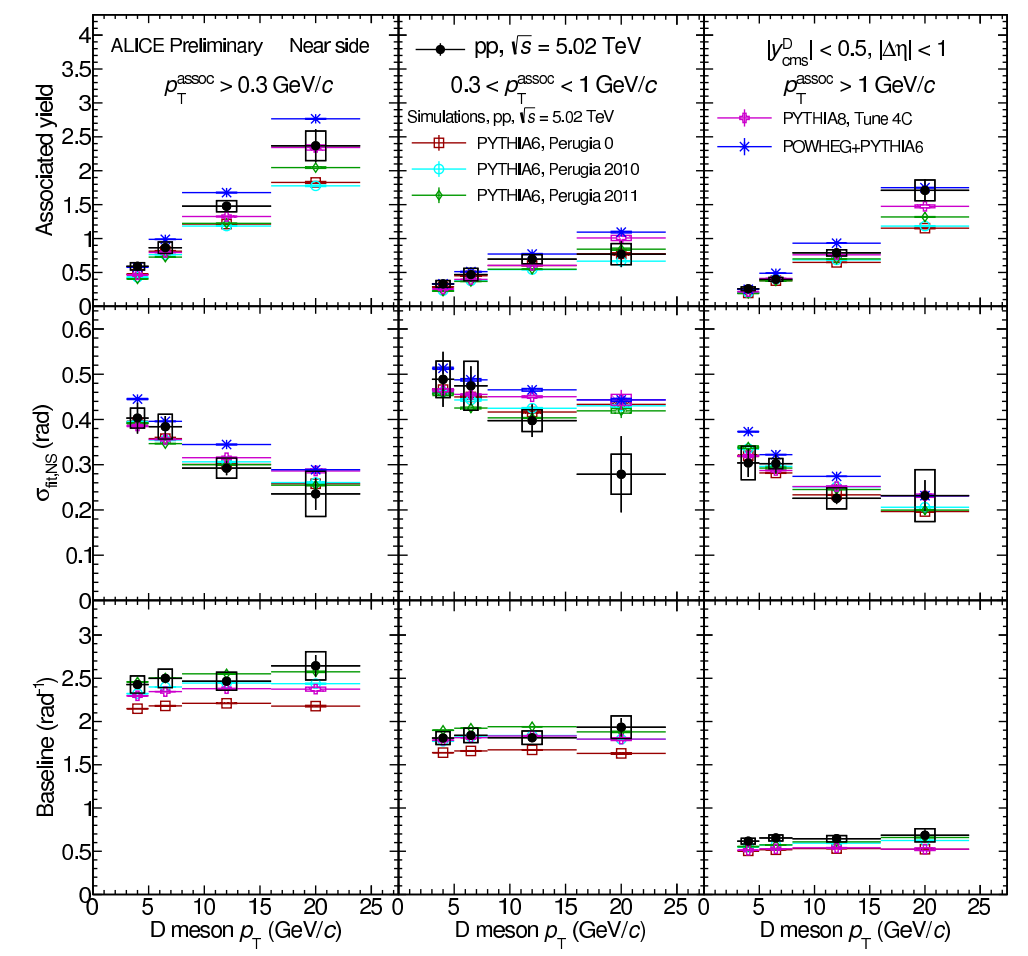

ALI-PREL-307362

Figure 4: Comparison of the near-side yields (top) and widths (mid) and baseline (bottom) data with model predictions in pp collisions at $\sqrt{s}=5.02 \mathrm{TeV}$.

of modifications due to the presence of cold nuclear matter effects. The near-side observables are compatible with model predictions.

\section{References}

[1] Aamodt, K. et al. [ALICE Collaboration], JINST 3 (2008)

[2] B. B. Abelev et al. [ALICE Collaboration], Int. J. Mod. Phys. A 29 (2014) 1430044 doi:10.1142/S0217751X14300440 [arXiv:1402.4476 [nucl-ex]].

[3] J. Adam et al. [ALICE Collaboration], Phys. Lett. B 754 (2016) 81 doi:10.1016/j.physletb.2015.12.067 [arXiv:1509.07491 [nucl-ex]].

[4] M. Cacciari, G. P. Salam and G. Soyez, JHEP 0804 (2008) 063 doi:10.1088/1126-6708/2008/04/063 [arXiv:0802.1189 [hep-ph]].

[5] M. Cacciari, G. P. Salam and G. Soyez, Eur. Phys. J. C 72 (2012) 1896 doi:10.1140/epjc/s10052-012-1896-2 [arXiv:1111.6097 [hep-ph]].

[6] B. Abelev et al. [ALICE Collaboration], JHEP 1203 (2012) 053 doi:10.1007/JHEP03(2012)053 [arXiv:1201.2423 [hep-ex]].

[7] S. Frixione, P. Nason and C. Oleari, JHEP 0711 (2007) 070 doi:10.1088/1126-6708/2007/11/070 [arXiv:0709.2092 [hep-ph]]. 
[8] T. Sjostrand, S. Mrenna and P. Z. Skands, JHEP 0605 (2006) 026 doi:10.1088/1126-6708/2006/05/026 [hep-ph/0603175].

[9] S. Acharya et al. [ALICE Collaboration], arXiv:1905.02510 [nucl-ex].

[10] J. Adam et al. [ALICE Collaboration], Eur. Phys. J. C 77 (2017) no.4, 245 doi:10.1140/epjc/s10052-017-4779-8 [arXiv:1605.06963 [nucl-ex]].

[11] T. Sjostrand, S. Mrenna and P. Z. Skands, Comput. Phys. Commun. 178 (2008) 852 doi:10.1016/j.cpc.2008.01.036 [arXiv:0710.3820 [hep-ph]]. 